\title{
Live Measurement of Electrical Charge Density in Materials using Off-Axis Electron Holography
}

\author{
E. Voelk1 ${ }^{1}$, F. Zheng ${ }^{2}$, V. Migunov ${ }^{2,3}$, M. Beleggia ${ }^{4}$ and R.E. Dunin-Borkowski ${ }^{2}$ \\ ${ }^{1}$ HoloWerk LLC, Austin, Texas \\ ${ }^{2}$ Ernst Ruska-Centre for Microscopy and Spectroscopy with Electrons, Forschungszentrum Jülich \\ GmbH, Jülich, Germany \\ ${ }^{3}$ Central Facility for Electron Microscopy, RWTH Aachen University, Aachen, Germany \\ ${ }^{4}$ DTU Nanolab, Technical University of Denmark, Kgs. Lyngby, Denmark
}

Over seventy years have elapsed since the invention of electron holography by Gabor [1]. However, it is only now that electron holography, and specifically its off-axis configuration, is becoming a mainstream technique. Many of the reasons for the slow development and implementation of the technique are related to the requirements for the experimental acquisition and analysis of electron holograms [2]. The recent introduction of single electron detection cameras and the emerging availability of parallel processing on computers are providing an environment that allows not only the use of off-axis electron holography at the microscope for live phase imaging but also the evaluation of recorded data on the fly.

Typically, image acquisition and analysis at the electron microscope is performed using software provided by the camera and/or microscope manufacturers. In addition to basic data processing capabilities, most commercial software also includes capabilities for specialized data analysis. For example, GMS software from Gatan [3] includes a proprietary scripting language that can be used to expand its analytical capabilities beyond those offered in its basic configuration. However, a proprietary scripting language is often limited in the range of operations that can be carried out. In addition, low level scripting is usually inaccessible to the average user. As a result, data processing is typically performed offline using other data analysis software, such as MATLAB. However, such an approach prevents live in situ data processing, analysis and interpretation, slowing down work and research.

Here, we describe the implementation of a capability to profile charge distributions in materials live in the same GMS software environment that is used for image acquisition. A complication arises if charge distributions are desired from rectangular areas that are not aligned with the conventional $x$ and $y$ axes of the camera, as standard region of interest tools in GMS are non-rotatable. However, the line profile tool in GMS can be manipulated using scripting to allow the selection of an area $D$, from which a charge distribution can be computed at any angle. Figure 1 shows the use of this line profile tool to plot charge distributions, whose computation can be summarized as follows. Electron optical phase images recorded using off-axis electron holography are sensitive to the projected electrostatic potential within and around a charged object, as described in detail elsewhere (e.g., [4-6]). The electrostatic contribution to the phase shift of a high energy electron wave $\psi(x, y)$ recorded using electron holography is given by the expression $\varphi(x, y)=C_{E} \int V(x, y, z) \mathrm{d} z$, where $\varphi(x, y)$ is the phase of the electron wave, $V(x, y, z)$ is the electrostatic potential within and around the specimen and $C_{E}$ is a constant that depends on the microscope accelerating voltage. The charge $Q_{D}$ in an area $D$ of the phase image can be measured either by evaluating the line integral of the gradient of the phase $-\varepsilon_{0} / C_{E} \oint \nabla \varphi(x, y) \mathbf{n} \mathrm{d} s$ or by evaluating the Laplacian of the phase $-\varepsilon_{0} / \mathrm{C}_{\mathrm{E}} \iint \nabla^{2} \varphi(x, y) \mathrm{d} x \mathrm{~d} y$. By using the identity $\nabla^{2} \varphi=\operatorname{Im}\left[\nabla^{2} \psi / \psi-(\nabla \psi)^{2} / \psi^{2}\right], Q_{D}$ can be obtained directly from the complex wave $\psi(x, y)$, thereby avoiding complications resulting from phase unwrapping. Based on these approaches, charge distribution profiles were obtained live using both 
the Laplacian method and the gradient method from phase images of $\mathrm{MgO}$ cubes, as shown in Fig. 1. In the present example, the charge density is affected by contributions from effective dipole layers resulting from local changes in mean inner potential and sample thickness at the edges of the cubes. The use of the complex image wave $\psi(x, y)$ for computing the charge distribution directly appears to be less prone to artefacts from large phase jumps. However, we are still investigating numerical artefacts.

It should be noted that single electron detection provides a great signal/noise-ratio advantage for electron holography. In the present study, off-axis electron holograms were recorded using a Gatan K2 camera $(3696 \times 3832$ pixels $)$ with a binning factor of 4 on an FEI Titan microscope operated at $300 \mathrm{kV}$. For live reconstruction, processing and visualization of the charge distribution, HoloWorks v6 software featuring multi-core processing was used on a dual Intel Xeon E5 2690 0, 2.90GHz computer. The computer screen was recorded using a smartphone and the resulting video was evaluated using the software Camtasia to determine the update rate for the charge distribution profiles. We are currently able to obtain $>2$ profiles per second during live charge density observation.

\section{References:}

[1] D. Gabor, Nature 161(4098) (1948) 777-78.

[2] E. Voelkl, Microscopy Today, 2019 January, DOI:10.1017/S1551929518001074.

[3] See http://www.gatan.com/

[4] M. Beleggia et al, Applied Physics Letters 98, 243101 (2011).

[5] V. Migunov et al, Journal of Applied Physics 117, 134301 (2015).

[6] M. Beleggia et al, 2016 J. Phys. D: Appl. Phys. 49294003.

[7] The authors acknowledge the European Union for funding through the Marie Curie Initial Training Network Grant No. 606988 under FP7-PEOPLE-2013-ITN).

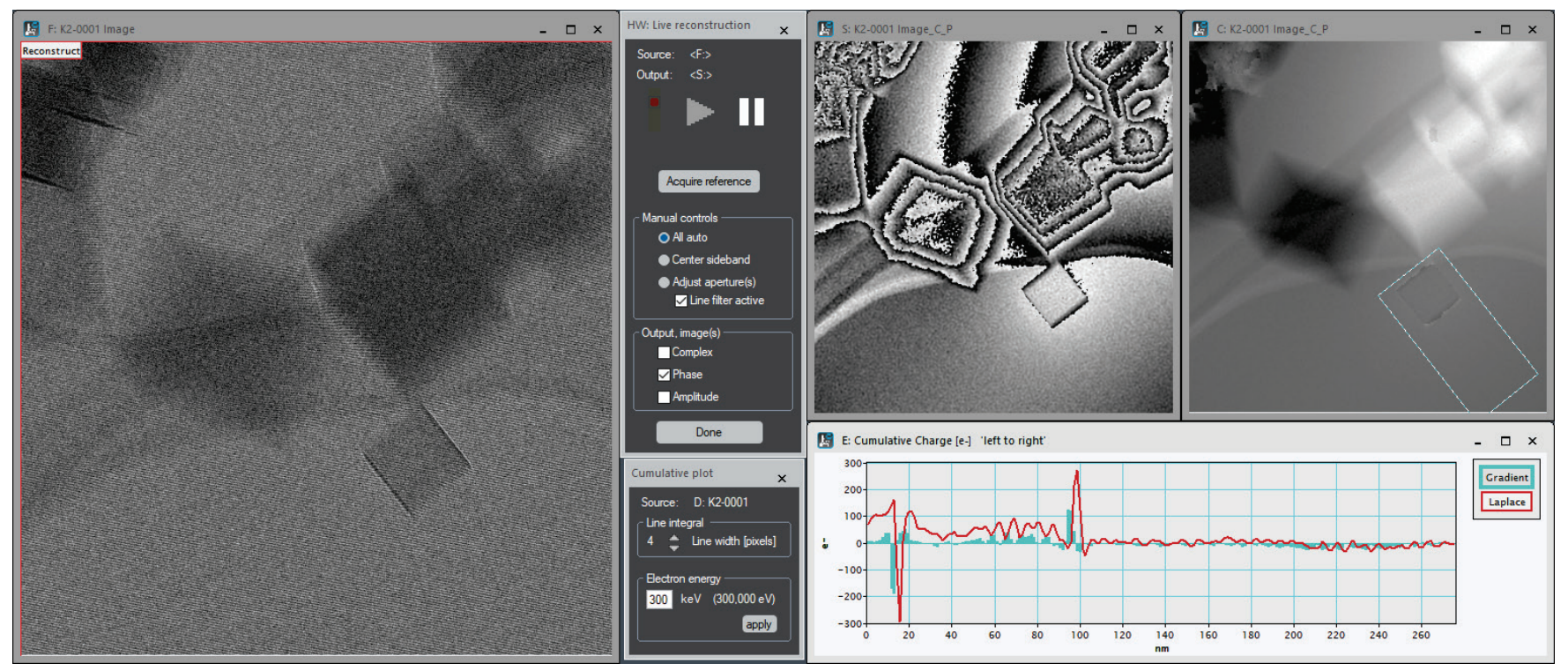

Figure 1. Screenshot showing setup for live charge density profiling from the indicated area in an unwrapped phase image (right). Left: live hologram. Top/middle: reconstructed phase. A typical speed of $>2$ profiles /second allows observation of changes in charge density profile at the microscope. The speed is limited mainly by the CPU, phase unwrapping and scripting overhead. Exposure time: $0.1 \mathrm{~s}$. 